\title{
A 1-metre Ni coated CFRP demonstrator for large deformable mirrors
}

\author{
Samantha J. Thompson ${ }^{1, \text { a }}$, Peter Doel ${ }^{1, \mathrm{~b}}$, David Brooks ${ }^{1}$, and Martin Strangwood ${ }^{2}$ \\ 1 Physics and Astronomy Department, University College London, Gower Street, London, WC1E \\ 6BT, UK \\ 2 Metallurgy and Materials, The University of Birmingham, Edgbaston, Birmingham, B15 2TT, UK
}

\begin{abstract}
We present results from our current project to develop an alternative substrate for large deformable mirrors, particularly with the European Extremely Large Telescope (E-ELT) in mind. Our mirror substrate consists of a carbon-fibre reinforced polymer (CFRP) core encapsulated in a thick $(50 \mu \mathrm{m})$ coating of nickel; the coating entirely covers the CFRP front, back and edges. The benefits of CFRP are: that it has high tensile strength, making it exceptionally resistant to breakage and able to withstand high inter-actuator forces; that it can be fabricated in large sections, allowing the production of a $2.6 \mathrm{~m}$ monolithic mirror, simplifying system control and eliminating additional diffraction/scattering introduced by segmented mirror systems; its low density $\left(<1800 \mathrm{kgm}^{-3}\right.$ for a $\mathrm{Ni}$ coated substrate). By the end of summer this year (2009) we aim to have constructed a $19 \mathrm{~cm}$ diameter fully actuated (37 piezo-stack actuators on a $29 \mathrm{~mm}$ triangular grid) prototype and a $1.0 \mathrm{~m}$ diameter substrate mounted on a static set of points to demonstrate the scalability of the technology. We discuss the processes involved in forming a Ni-CFRP mirror, the results obtained so far and a current status update.
\end{abstract}

\section{Introduction}

The design studies for next generation ground-based optical telescopes are underway. A number of these (e.g. the Giant Magellan Telescope (GMT) [1] and the European Extremely Large Telescope (EELT) [2]) are planning to incorporate a large adaptive mirror as an integrated part of the telescope. Of particular relevance to this research is the adaptive M4 in the E-ELT design which is a $2.6 \mathrm{~m}$ diameter flat; current thin glass shell technology has so far been unable to demonstrate scalability to this size for a monolithic mirror and segmented designs pose other problems related to scattered light and unwanted diffraction effects as well as increasing the operational complexity. The GMT design has alleviated this problem by having the same number of adaptive secondaries as there are primary segments and mapping each primary segment to a particular adaptive mirror of a size that is within manufacturing range for thin glass mirrors. For designs requiring larger adaptive mirrors or where fracture of thin glass plates is of concern, carbon- fibre composite (CFC) mirrors are a possible solution. Carbon-fibre mirrors can be useful where weight is an issue, both in space and ground based telescopes. CFC can be made to large diameters (limited only by the size of the replication mandrel that can be manufactured, since out-of-autoclave curing methods are now available), are lightweight, robust and can be handled and cleaned without risk of breakage. Carbon-fibre is available with a wide range of properties and can be combined with a variety of different resin systems to provide a material tailored to requirements.

Carbon-fibre has been used for a number of years to construct the panels in radar dishes, communications satellites and other longer wavelength (down to sub-millimeter) telescope apertures. So far CFC mirrors have not been used for visible wavelength imaging due to surface quality issues and low form accuracy. The Optical Science Laboratory at UCL has been investigating different types of carbon-fibre composite mirror for use in optical applications for over 5 years. Initial prototypes were stiffer, actively controlled mirrors composed of an electroformed nickel face-plate bonded to a carbonfibre-aluminium honeycomb sandwich [3,4]. More recent developments have been towards adaptive mirrors made from thin carbon composite cores and electroplated in nickel to provide the reflective surface $[5,6]$.

\footnotetext{
a e-mail: sjt@star.ucl.ac.uk

b e-mail: apd@star.ucl.ac.uk
} the original work is properly cited. 
The aims of our current project are to manufacture, coat and polish: (1) a $19 \mathrm{~cm}$ diameter nickel coated carbon-fibre composite (Ni-CFC) mirror mounted to a bed of 37 actuators on a $29 \mathrm{~mm}$ diameter triangular grid spacing, (2) a $1.0 \mathrm{~m}$ diameter Ni-CFC mirror. Also included will be a analysis of the material properties of the Ni-CFC and the reproducibility and consistency of material properties over different manufacturing runs. The first aim will demonstrate the suitability of the material in use as an adaptive optic, including a lifetime actuation test and comparisions with simulated results; the second aim will demonstrate the scalability of the technology, due to budget constraints this large mirror will either be left unmounted or on a mount that allows some manual adjustment of the mirror form. Here we present an overview of the methods used to make the Ni-CFC mirrors and the recent progress of the project. A more detailed description of the methods and materials analyses in this project can be found in previous papers $[6,7]$.

\section{Nickel-Carbon-Fibre-Composite mirrors}

The manufacture of a Nickel-Carbon-Fibre-Composite (Ni-CFC) mirror involves 4 key stages: (1) making an appropriate mandrel for the CFRP replication process, (2) CFRP lay-up and curing, (3) nickel electroplating and (4) optical polishing.

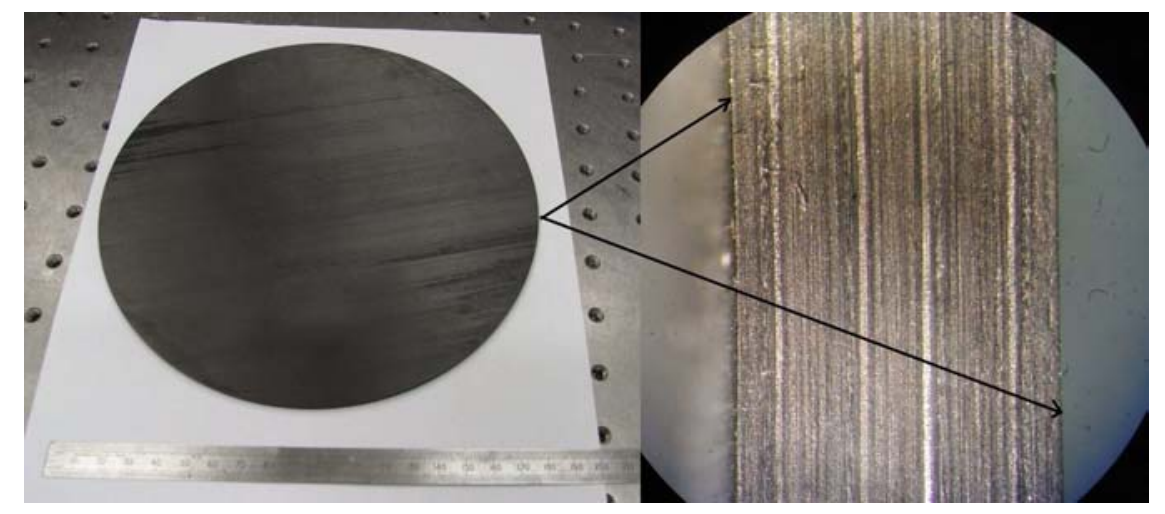

Fig. 1. The photograph shows the CFRP core as prepared before the nickel coating procedure. The polished edge as viewed under a microscope shows the symmetric stack of 48 plies of unidirectional CFRP material.

\subsection{Mandrel fabrication}

The carbon-fibre mirror core is made via a replication process. A mould or mandrel of the inverse required mirror form is first manufactured and the carbon-fibre plies are placed on to this mould. For this project we are manufacturing flat mirrors, as this is the form chosen for the M4 adaptive mirror in the ESO ELT design. We therefore require flat mandrels. In past projects we have also made spherical, concave mirrors, requiring the more difficult to measure, convex mandrels.

We use slightly oversized mandrels and the CFRP core is trimmed down to the required mirror diameter, this allows the removal of any edge effects which can sometimes occur during the CFRP replication process. Since we are producing flat moulds/mirrors we have chosen to make the mandrels out of an aluminium alloy, however other mirrors forms would require the use of a low thermal expansion material to ensure that the mandrel did not deform to an unacceptable degree during the curing process. A $1.05 \mathrm{~m}$ diameter moulding tool has been finished to a few microns surface flatness and a fine ground surface finish. The CFRP mirror core will be trimmed to a $1.0 \mathrm{~m}$ diameter before the coating procedure. 


\subsection{Carbon-fibre reinforced polymer}

The core of the Ni-CFC mirror is made from sheets of a unidirectional carbon- fibre impregnated with a resin. The CFRP core is made via a replication process; the sheets (plies) are arranged in a stack in a specific angular sequence over a moulding tool which is then vacuum bagged and autoclaved to cure the resin.

We are using IM7 carbon-fibre (Hexcel) and LTM123 (Advanced Composites) cyanate ester resin in a $60 \%$ fibre volume ratio. IM7 is a mid-modulus fibre, allowing the production of a mirror with a Young's modulus very similar to that of glass $(70 \mathrm{GPa})$. Stiffer material can be made using higher modulus fibres (such as M55J by Toray), however for an adaptive optics application this would then require stiffer, higher force actuators which tend to be more expensive and larger in bulk; we are aiming to minimise the mass of the system. Our lay-up sequence uses a $15^{\circ}$ angular increment between $0 / 90$ ply-pairs and has 2 pattern repeats either side of the centre symmetry plane, giving a total of 48 plies; Fig. 1 shows an example of a $19 \mathrm{~cm}$ diameter carbon-fibre plate and a microscope image of the cross-section showing the ply-stack. Inaccurate placement of plies can result in large scale form deformations due to unbalanced strains in the material on curing, these are minimised by placing the plies to an angular accuracy of 0.5 degrees or better. Each ply is $70 \mu \mathrm{m}$ in thickness, resulting in a finished mirror thickness of $3.3 \mathrm{~mm}$ after curing. The resin is fully cured in an autoclave (at $\mathrm{T} \sim 180^{\circ}$ ), this ensures that the resin gains its lowest moisture absorption properties. The final mirror thickness is dependent on the cure process and so to obtain consistent results the exact same schedule must be used each time.

\subsection{Nickel electro-chemical deposition}

There have been a number of issues regarding the use of CFRP material in optical quality mirrors. Firstly there is the environmental stability of the resin, primarily these are long term degradation due to exposure to UV radiation and absorption of water causing the material to expand and deform. These can be minimised to an extent by chosing a resin system such as the LTM123 toughened cyanate ester, it has excellent resistance to microcracking, one of the lowest moisture uptakes available and is NASA approved for space applications. The second issue is that of fibre print-through affecting the optical surface quality which is usually seen in thin reflective coatings over CFRP. To address all these issues we encapsulate the CFRP core in a thick $(\sim 50 \mu \mathrm{m})$ layer of nickel. Nickel is a metal which can be optically polished to sub-nm suface quality if required and has no known stable carbides, reducing the likelihood of any corrosion at the metal-CFRP boundary.

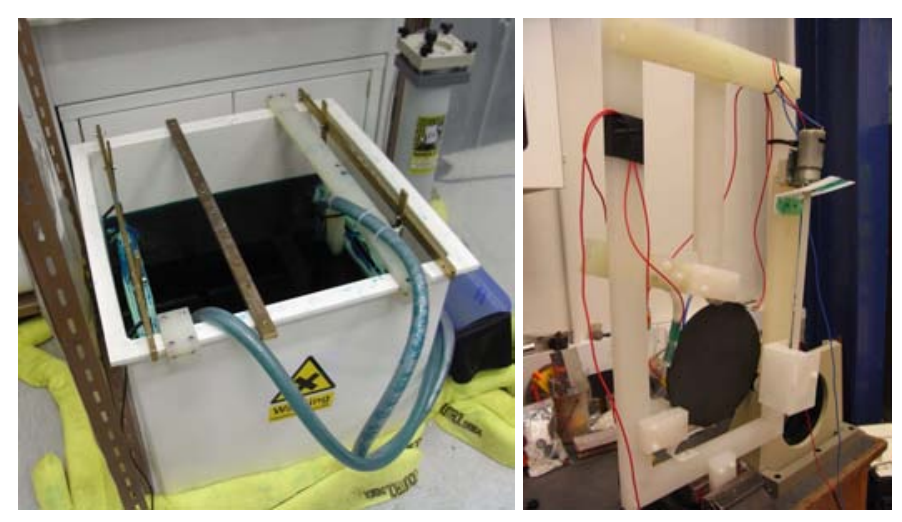

Fig. 2. The in-house nickel electroplating facility and the spinning electrode rig for coating the smaller mirrors.

The coating is applied in a nickel sulphamate electrochemical bath as shown in Fig. 2. The coating is applied to both sides and around the edges at the same time by means of a moving electrical contact, 
achieved by spinning the mirror in a motorised contraption (Fig. 2). The method was developed inhouse to provide a coating with low internal stresses, allowing subsequent grinding and polishing of the nickel layer so that minimal distortion occurs on removal of the material.

\subsection{Optical polishing and testing}

The nickel coating obtained using the electrochemical deposition needs to be ground and polished to produce the desired reflective, optical surface quality. Nickel can be polished to sub-nm surface roughness and can be thin-film coated, as for glass, with an appropriate reflection coating (e.g. gold) if required.
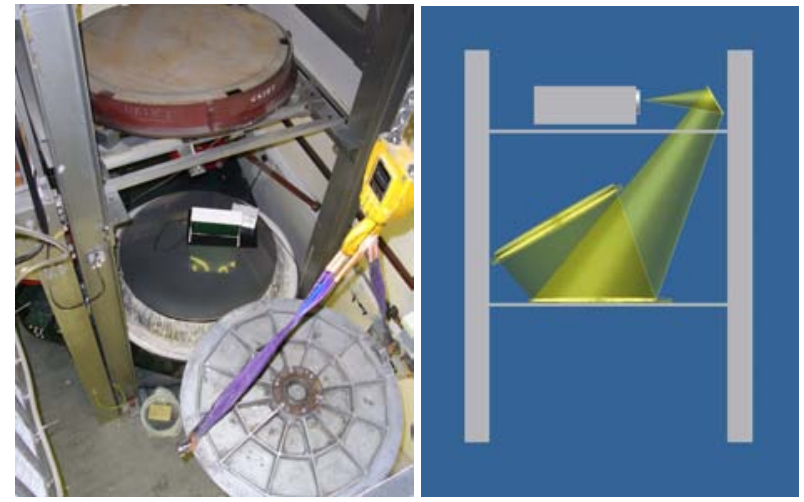

Fig. 3. The $1.05 \mathrm{~m}$ mandrel being polished on the large polishing table in the test tower (left); optical ray trace of a $1 \mathrm{~m}$ flat mirror tested against a reference sphere $(\mathrm{R}=2744 \mathrm{~mm}$, diameter $=1066 \mathrm{~mm})$ and overlaid on a CAD drawing of the test tower (left).

Due to the thin and flexible nature of the mirrors care must be taken during the polishing process to ensure an unwanted form of stress-polishing does not occur. Metrology for the $1.0 \mathrm{~m}$ mirror will be performed in a test tower using a large reference sphere and $633 \mathrm{~nm}$ Fizeau interferometer, the configuration is shown in Fig. 3.

\section{Finite element analysis}

Finite element analysis (FEA) has been utilised for a number of the processes involved in the creation of the Ni-CFRP mirror. These include optimisation of the mechanical design, improvements to the electroplating process and investigations into cure-cycle problems during the CFRP manufacturing. Figure 4 shows some example results, including: (1) a simulation to investigate an actuator influence function, a whole set of simulated functions are obtained and used to calculate the mirror modes; (2) the surface distortions on the mirror due to gravity on the $19 \mathrm{~cm}$ diameter prototype supported on 3 points on the Cer-Vit backing plate, parameters and supports can be optimised to minimise the surface effects.

The simulated influence functions and other system properties will be verified against the prototype during future testing.

\section{Current status and future work}

A mechanical and thermal properties analysis has been undertaken at the University of Birmingham. The measurements agree well with laminate theory; for this material we have a Young's Modulus 

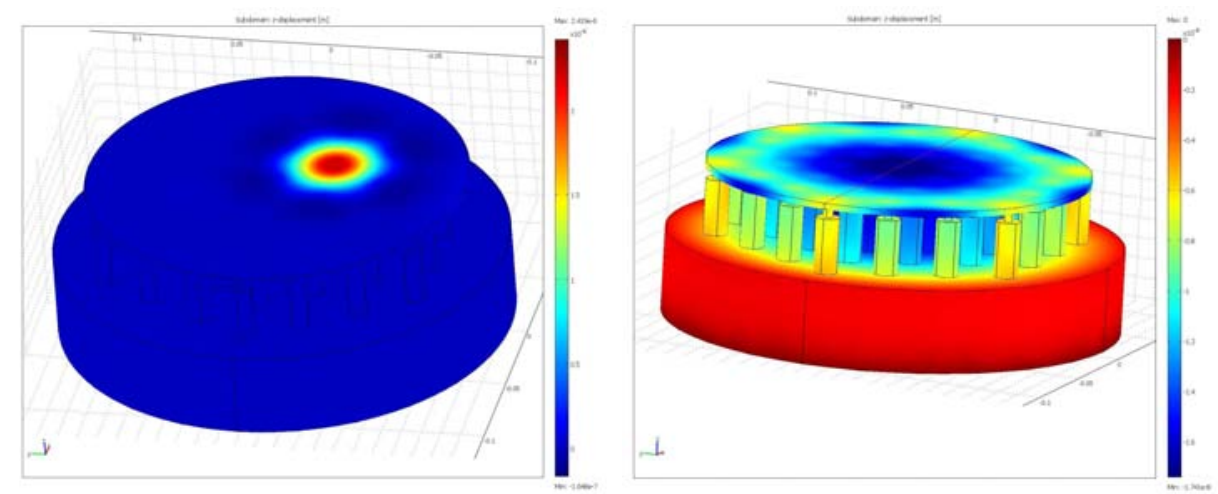

Fig. 4. Some FEA results: (left) an actuator influence function, (right) distortion of surface due to gravity - max surface $\mathrm{P}-\mathrm{V}$ for negative gravity vector is $10 \mathrm{~nm}$, this increases to $22 \mathrm{~nm}$ for the positive vector, assembly fixed by a 3-point support on rear of backing plate.

of $\sim 70 \mathrm{GPa}$ and a thermal expansion of $\sim 2 \mathrm{ppm}$. Mirror samples have undergone multiple cycles of loading and unloading (up to $20 \mathrm{~kg}$ ) and have displayed linear elastic behaviour throughout. Some additional testing is still ongoing and the $1.0 \mathrm{~m}$ CFRP core will be non- destructively tested (ultrasound investigations and vibration analysis) to check the material properties over this much larger surface area than has previously been investigated with the $19 \mathrm{~cm}$ diameter samples.
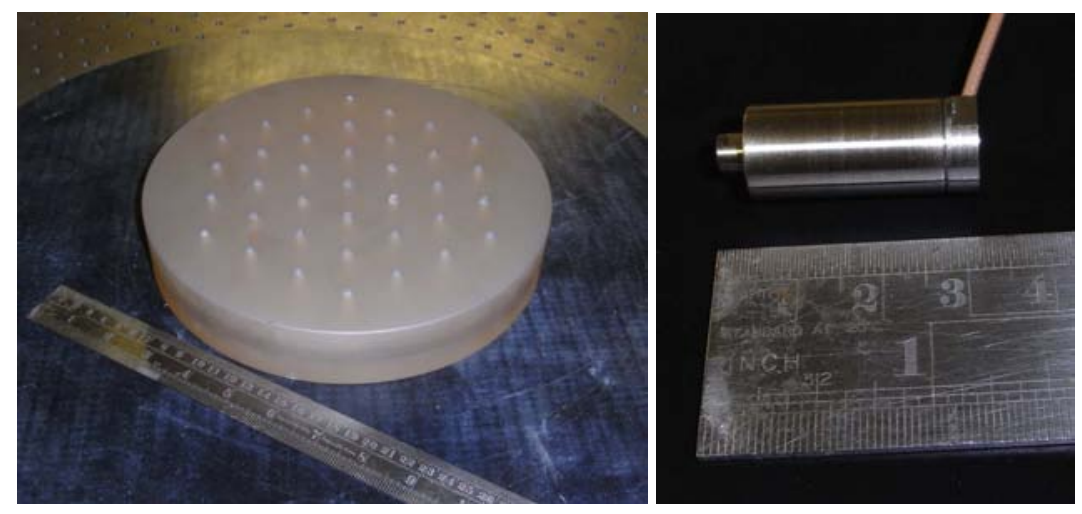

Fig. 5. The $23 \mathrm{~cm}$ diameter cervit backing plate, ground flat and drilled to mount the piezo column actuators (shown right).

The $19 \mathrm{~cm}$ diameter prototype is under construction. The backing plate will consist of a Cer-Vit flat (zero expansion ceramic) on which the 37 piezo stack column actuators will be mounted (see Fig. 5). The actuators are a custom cased (in aluminium to reduce mass) and custom pre-loaded piezo-stack provided by PiezoMechanik. Each actuator has a mass of only $\sim 8 \mathrm{~g}$ (not including cabling), is $30 \mathrm{~mm}$ in height and provides an average of $12 \mu \mathrm{m}$ displacement over a $0-150 \mathrm{~V}$ range when coupled to the mirror. The actuators will be coupled to the Ni-CFRP mirror via small metal flexures. The actuators will be driven via custom drive electronics (StarpointAO).

The first $1.0 \mathrm{~m}$ CFRP plate to be produced from the $1.05 \mathrm{~m}$ mould has just been manufactured, photographs of it coming out of the autoclave are shown in Fig. 6. This plate is a thinner lay-up than the ones that will go on and be fully polished; this first run was used to practice lay-up techniques and handling of the larger ply cut-outs to minimise fibre distortions, check for any problems during 

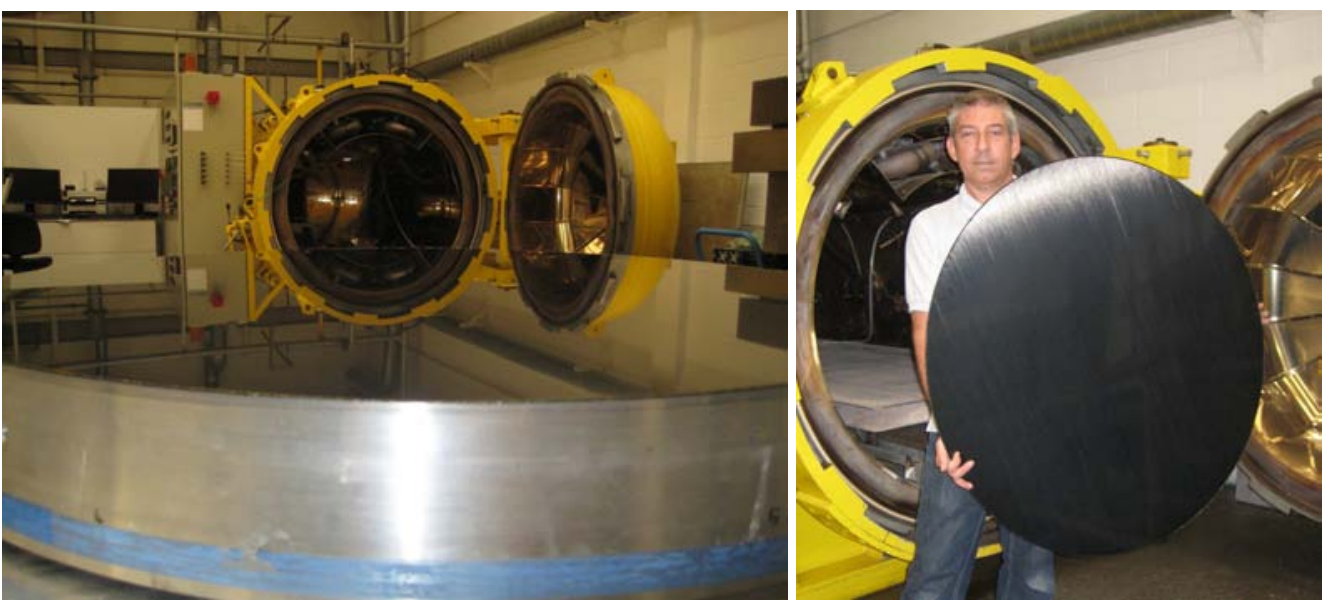

Fig. 6. The first $1.0 \mathrm{~m}$ CFRP mirror production run; (left) just removed from the autoclave and still seated on the mould, (right) released from the mould.

the cure schedule and test out the $1.0 \mathrm{~m}$ electroplating facility which is being setup by WaveForm Ltd. The full 48 ply lay-up on the $1.05 \mathrm{~m}$ mould is planned shortly and we anticipate a completed $1.0 \mathrm{~m}$ Ni-CFRP mirror by the end of this year.

\section{Acknowledgements}

We would like to thank the various companies we have worked with that have offered us useful advice and discussions; Hart Coating Technology and Waveform Ltd. in electroplating, and B3 Technologies (now dissolved) and FormTech Composites in CFRP production.

This work is funded by the Science and Technology Facilities Council (STFC) under the guidance of the UK ELT Steering Committee.

\section{References}

1. M. Lloyd-Hart and R. Angel and N. M. Milton and M. Rademacher and J. Codona, Proc. SPIE 6272, (2006)

2. Gilmozzi, R. and Spyromilio, J., The Messenger 127, (2007) pp. 11-19

3. Doel, A. P. and Kendrew, S. and Brooks, D. and Dorn, C. and Yates, C. and Dwan, R. M. and Richardson, I. and Evans, G., Proc. SPIE 5490, (2004) pp. 1526-1533

4. Kendrew, S. and Doel, P. and Brooks, D. and King, A. M. and Dorn, C. and Yates, C. and Dwan, R. M. and Richardson, I. M. and Evans, G., Optical Engineering 46, (2007)

5. Samantha J. Thompson and Peter Doel and David Brooks, Adaptive Optics: Analysis and Methods/Computational Optical Sensing and Imaging/Information Photonics/Signal Recovery and Synthesis Topical Meetings on CD-ROM (Optical Society of America, 2007) pp. ATuD5

6. S. J. Thompson and D. Brooks and A. P. Doel, Optics Express 16, (2008) pp. 1321-1330

7. S. J. Thompson, A. P. Doel, D. Brooks, M. Strangwood, Proc. SPIE 7018, (2008) pp. 701839701839-10 